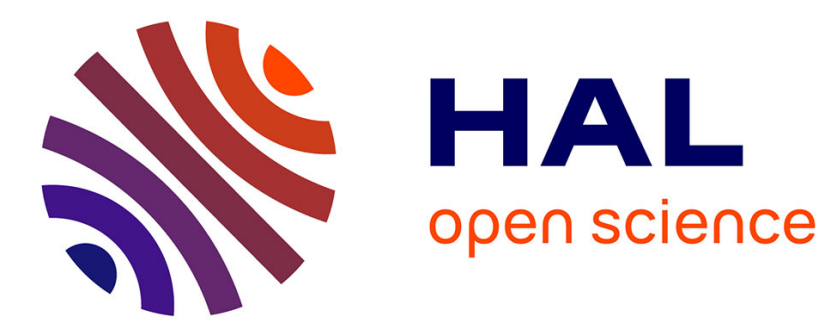

\title{
Ultrasonic investigation of critical behaviour and percolation phenomena in microemulsions
}

\author{
R. Zana, Jérôme Lang, O. Sorba, A.M. Cazabat, D. Langevin
}

\section{To cite this version:}

R. Zana, Jérôme Lang, O. Sorba, A.M. Cazabat, D. Langevin. Ultrasonic investigation of critical behaviour and percolation phenomena in microemulsions. Journal de Physique Lettres, 1982, 43 (23), pp.829-837. 10.1051/jphyslet:019820043023082900 . jpa-00232132

\section{HAL Id: jpa-00232132 https://hal.science/jpa-00232132}

Submitted on 1 Jan 1982

HAL is a multi-disciplinary open access archive for the deposit and dissemination of scientific research documents, whether they are published or not. The documents may come from teaching and research institutions in France or abroad, or from public or private research centers.
L'archive ouverte pluridisciplinaire HAL, est destinée au dépôt et à la diffusion de documents scientifiques de niveau recherche, publiés ou non, émanant des établissements d'enseignement et de recherche français ou étrangers, des laboratoires publics ou privés. 
Classification

Physics Abstracts

$43.35-64.70 \mathrm{~J}-68.10 \mathrm{C}-82.70$

\title{
Ultrasonic investigation of critical behaviour and percolation phenomena in microemulsions
}

\author{
R. Zana, J. Lang \\ Centre de Recherches sur les Macromolécules, 6, rue Boussingault, 67083 Strasbourg, France
}

O. Sorba, A. M. Cazabat and D. Langevin

Laboratoire de Spectroscopie Hertzienne de l'E.N.S., 24, rue Lhomond, 75231 Paris Cedex 05, France

(Reçu le 9 août 1982, accepté le 4 octobre 1982)

\begin{abstract}
Résumé. - Plusieurs systèmes micellaires qui, étudiés par diffusion de la lumière, présentent un comportement critique ont été étudiés à l'aide de techniques d'absorption ultra-sonore. Un excès d'absorption ultra-sonore a été observé dans les systèmes à phase continue organique, pour lesquels le " point critique " se trouve près d'un seuil de percolation. Dans les systèmes à phase continue aqueuse, on n'observe pas d'augmentation de l'absorption, même près du " point critique ". On en conclut que l'origine de l'augmentation de l'absorption dans les systèmes à phase continue organique est plutôt due à l'apparition de structures ouvertes au seuil de percolation.
\end{abstract}

\begin{abstract}
Several micellar systems showing critical-like behaviour, as evidenced by light scattering measurements, have been investigated by ultrasonic absorption techniques. An anomalous increase in the ultrasonic absorption has been observed in oil-external systems where the "critical point " is close to a percolation threshold, whereas no increase of the absorption is seen in water-external systems even close to the " critical point ". It is concluded that the origin of the increase of absorption in oil-external systems is due rather to the appearance of open structures at the percolation threshold.
\end{abstract}

1. Introduction. - Microemulsions consist of water (or oil) droplets of fairly small diameter $(4-40 \mathrm{~nm})$ surrounded by a film of surfactant and cosurfactant (most generally alcohol) molecules, dispersed in a continuous organic or aqueous medium.

Recently, elastic and quasi-elastic light scattering studies have shown that some microemulsions behave like systems close to their critical points [1-5]. This finding may be of great importance for the understanding of the low interfacial tensions observed with microemulsions used for tertiary oil recovery.

However, the critical-like behaviour of microemulsion is far from being fully understood. In particular, the critical exponents depend on the nature of the constituents $[2,3]$. Similar observations have been recently reported for micellar solutions [6], which are binary mixtures of water and surfactant. This non-universal behaviour may arise from the unequal sizes of molecular and micellar entities. Moreover, the range of volume fraction where the critical-like behaviour for water-in-oil microemulsions is observed is also where a steep increase in the electrical conductivity occurs $[1,4,5]$. This last phenomenon has been attributed to percolation of the water droplets [7]. The relationship between critical-like behaviour and percolation in microemulsions is still unclear. 
The above observations call for further studies in relation to critical phenomena using other methods of investigation. This prompted us to study the ultrasonic absorption of various systems showing critical-like behaviour. The purpose of these experiments is readily understood by recalling that, for instance, the ultrasonic absorption of a binary liquid mixture goes through a maximum at the critical concentration. The amplitude of this maximum increases rapidly as the temperature of the mixture becomes closer to the critical temperature $T_{\mathrm{c}}$. At $T_{\mathrm{c}}$ the ultrasonic absorption diverges. This behaviour is due to the coupling between the temperature and pressure changes associated with the propagation of the ultrasonic waves through the system and the long-range fluctuations of composition occurring in the system as it is brought nearer to critical conditions [8-10].

2. Systems. - All the systems investigated in this work have been previously studied by means of light scattering $[1,2,3,6]$.

2.1 WATER-IN-OIL MICROEMUlSIONS ALONG A DILUTION LINE [1]. — In such systems, the concentration of droplets can be varied without changing their size and composition. We recall that the procedure of dilution involves microemulsions whose composition corresponds to points on a demixing line in the phase diagram. The criteria for a proper dilution is the linearity of the plot of the volume of added oil versus the volume of added alcohol + water, over a wide range of volumes [11]. The compositions of the continuous phases and of the droplets of the systems are given in table $\mathrm{I}$.

Table I.

Microemulsion

Droplets composition

Continuous phase composition

ATB

ATP

$\mathrm{BCP}$
$0.4 \mathrm{~g} \mathrm{SDS}$

$0.5 \mathrm{~cm}^{3}$ water

$0.17 \mathrm{~cm}^{3}$ butanol

$0.4 \mathrm{~g} \mathrm{SDS}$

$0.5 \mathrm{~cm}^{3}$ water

$0.33 \mathrm{~cm}^{3}$ pentanol

$0.4 \mathrm{~g}$ SDS

$1 \mathrm{~cm}^{3}$ water

$0.2 \mathrm{~cm}^{3}$ pentanol
$100 \mathrm{~cm}^{3}$ toluene

$13.5 \mathrm{~cm}^{3}$ butanol

$0.4 \mathrm{~cm}^{3}$ water

$100 \mathrm{~cm}^{3}$ toluene

$17 \mathrm{~cm}^{3}$ pentanol

$0.4 \mathrm{~cm}^{3}$ water

$100 \mathrm{~cm}^{3}$ cyclohexane

$19 \mathrm{~cm}^{3}$ pentanol

$0.32 \mathrm{~cm}^{3}$ water

2.1.1 Water-sodium dodecylsulfate (SDS)-toluene-butanol system (ATB $\left({ }^{1}\right)$ ) [12]. - The investigation of these microemulsions by means of light scattering as a function of the droplet volume fraction $\phi$ revealed a marked maximum of scattered intensity $I$ and a minimum of the droplet diffusion coefficient $D$ at $\phi \sim 0.1$ [1]. This behaviour, as well as the angular dependence of $I$ and $D$, can be explained using theories pertaining to critical phenomena. Moreover, a steep increase of the electrical conductivity was observed at the same volume fraction and attributed to percolation [1].

2.1.2 Water-SDS-toluene-pentanol system $(A T P)$. - The droplet radius* in this system is $44 \AA$, very close to the value of $42 \AA$ found for the ATB system. Nevertheless, the ATP micro-

$\left({ }^{1}\right)$ Code for our w/o microemulsions systems [12]

first letter : water/soap weight ratio in droplet

$\begin{array}{lll}\text { A } 1.25 & \text { B } 2.50\end{array}$

second letter: oil $\quad \mathrm{T}$ toluene $\mathrm{C}$ cyclohexane

third letter: alcohol B butanol $\quad P$ pentanol 
emulsions show only minor changes of $D$ and $I$ in the investigated range of volume fraction $(\phi<0.2)$. These changes are well accounted for in terms of the hard-sphere model for the droplets [12]. No steep variation of the electrical conductivity is observed.

2.1.3 Water-SDS-cyclohexane-pentanol system (system BCP). - In this system the droplets behave like slightly attractive spheres with a radius of $75 \AA$ [12]. Light scattering does not show critical-like behaviour, but a steep increase of the electrical conductivity is observed at $\phi \sim 0.14$ [7].

2.2 SYSTEMS OF CONSTANT COMPOSITION AS A FUNCTION OF TEMPERATURE. - All these systems have been investigated by other authors by means of light scattering techniques and showed critical-like behaviour.

2.2 .1 n-dodecylhexaoxyethyleneglycol monoether $\left(\mathrm{C}_{12} \mathrm{E}_{6}\right)$-water system. - This micellar solution is the simplest system investigated. Its critical concentration and temperature have been reported to be $1.25 \%$ (wt./vol.) and $50.3{ }^{\circ} \mathrm{C}$, respectively [6]. However, the investigated sample showed a cloud point at $38.5^{\circ} \mathrm{C}$ only, probably because of the presence of a long-chain impurity, most likely dodecylalcohol resulting from the hydrolysis of $\mathrm{C}_{12} \mathrm{E}_{6}$. Long-chain alcohols are known to considerably reduce the cloud point of non-ionic surfactants, by up to $45^{\circ} \mathrm{C}$ even at concentration as low as $0.5 \%$ [13]. However, the critical behaviour of the system, as evidenced by light scattering, was not affected.

2.2.2 Three components water-in-oil microemulsion [2] (AOT system). - A $6.3 \%$ (wt./vol.) sodium di-2-ethylhexylsulfosuccinate (AOT) solution in n-decane, containing $9 \%$ (wt./vol.) water (molar concentration ratio $\left[\mathrm{H}_{2} \mathrm{O}\right] /[\mathrm{AOT}]=35$ ), has been studied between $13.8^{\circ} \mathrm{C}$ and $37.8^{\circ} \mathrm{C}$. The demixtion temperature was $38.3^{\circ} \mathrm{C}$.

2.2.3 Five components oil-in-water microemulsion [3] (CTAB system). - The investigated systems contained (by wt. \%) $2.30 \%$ cetyltrimethylammoniumbromide (CTAB), $2.3 \%$ n-octane, $4.6 \%$ butanol, $1.7 \%$ sodium bromide, $89.1 \%$ water. It was found to show a critical-like behaviour at around $38{ }^{\circ} \mathrm{C}[3]$.

The systems ATB and ATP have been investigated at $20^{\circ} \mathrm{C}$ in the ultrasonic frequency range between 6.5 and $115 \mathrm{MHz}$. The absorption of the other systems was measured at frequencies between 4 and $22 \mathrm{MHz}$.

3. Experimental results and discussion. - The difference in behaviour between pentanol and butanol containing microemulsions is clearly apparent in figures 1 to 3 .

The ATP system shows a smooth change of the ultrasonic absorption $\alpha / f^{2}$ ( $\alpha=$ absorption coefficient in $\mathrm{cm}^{-1}, f=$ ultrasonic frequency in $\mathrm{Hz}$ ) with the droplet volume fraction $\phi$. On the contrary, at all the frequencies investigated the ATB system shows a maximum of absorption at $\phi \sim 0.1$.

Before discussing the mechanisms which may be responsible for the ultrasonic absorption of the ATP and ATB systems, we briefly recall the main conclusions of previous ultrasonic investigations of microemulsions $[14,15]$. For compositions far from the critical ones, the excess ultrasonic absorption of the system with respect to the continuous phase was essentially attributed to exchange processes. These processes refer to the exchange of alcohol and surfactant between the interfacial film and the continuous and/or dispersed phases. At low volume fraction, well below the phase-inversion range, such processes give rise to smooth $\alpha / f^{2} v s . \phi$ curves, such as those found for the ATP system $[14,15]$. An examination of the results of figure 3 then suggest that at low frequency the absorption of the ATB systems is made up of two contributions :

One contribution close to the absorption for the ATP system, evidenced by the fact that the $\alpha / f^{2} v s$. $\phi$ curves for the two systems are nearly coincident up to $\phi \sim 0.05$, and then again become very near at $\phi>0.2$ after diverging greatly in the range $0.05<\phi<0.2$. This contribution would be due to exchange processes. 


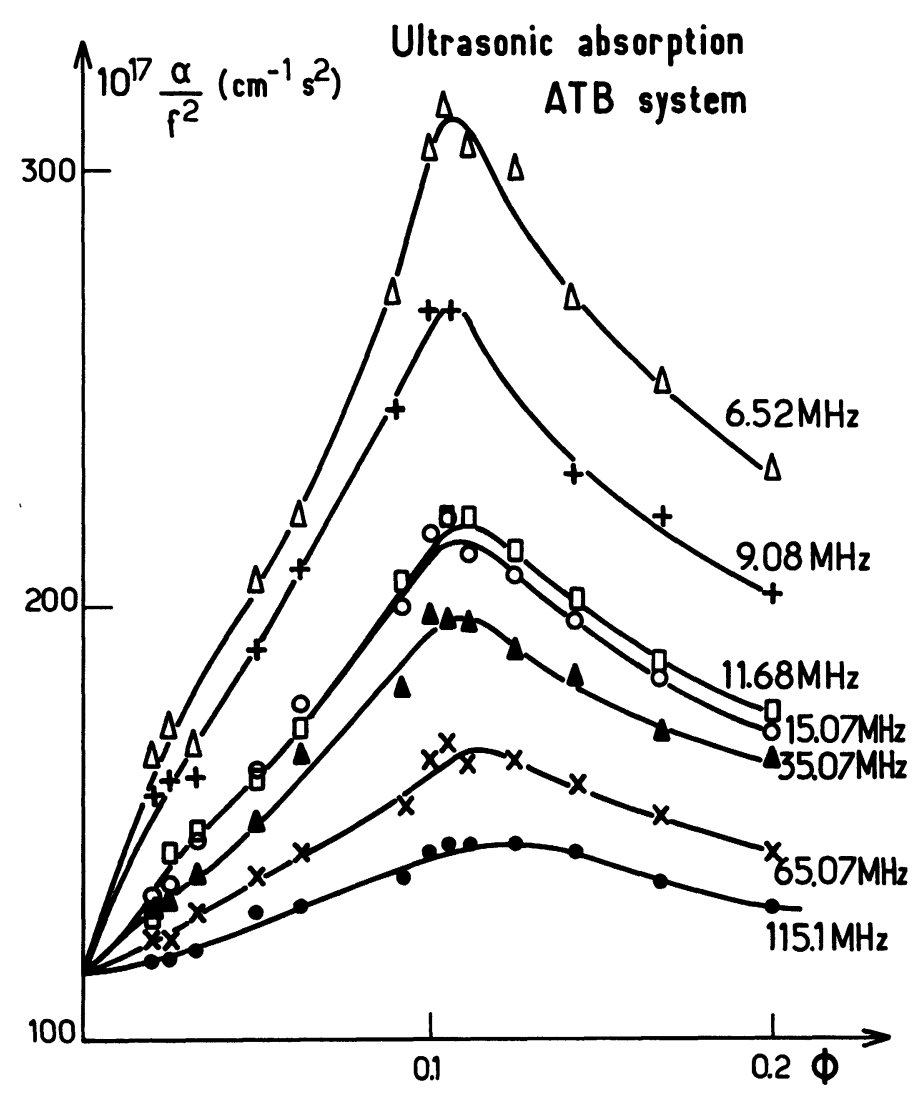

Fig. 1. - Ultrasonic absorption for ATB microemulsions versus volume fraction $\phi$ of droplets at different frequencies.

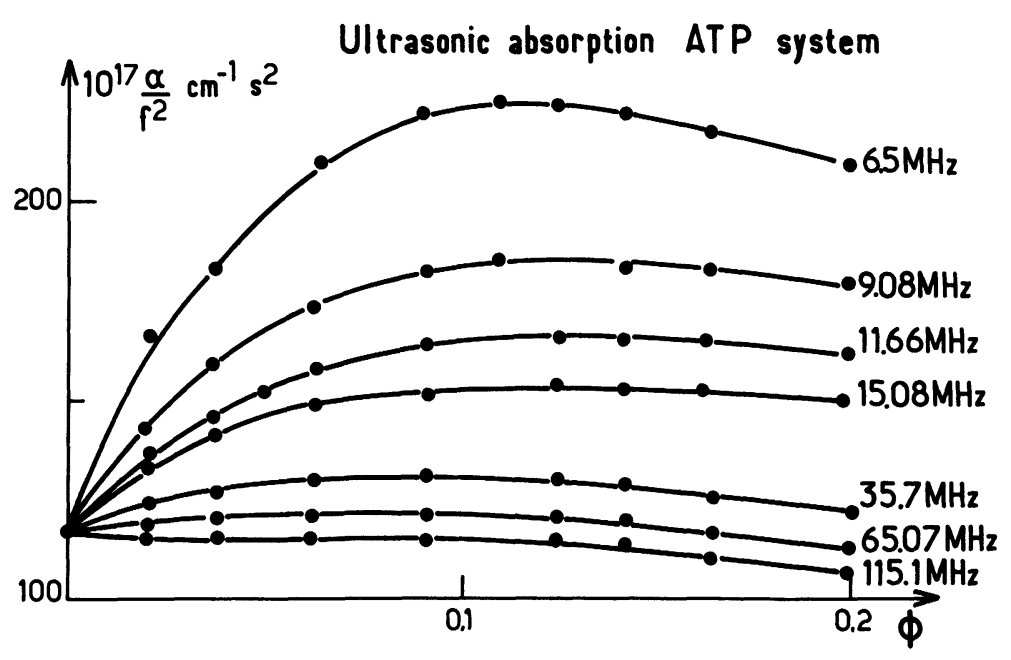

Fig. 2. - Ultrasonic absorption for ATP microemulsions versus volume fraction $\phi$ of droplets at different frequencies. 


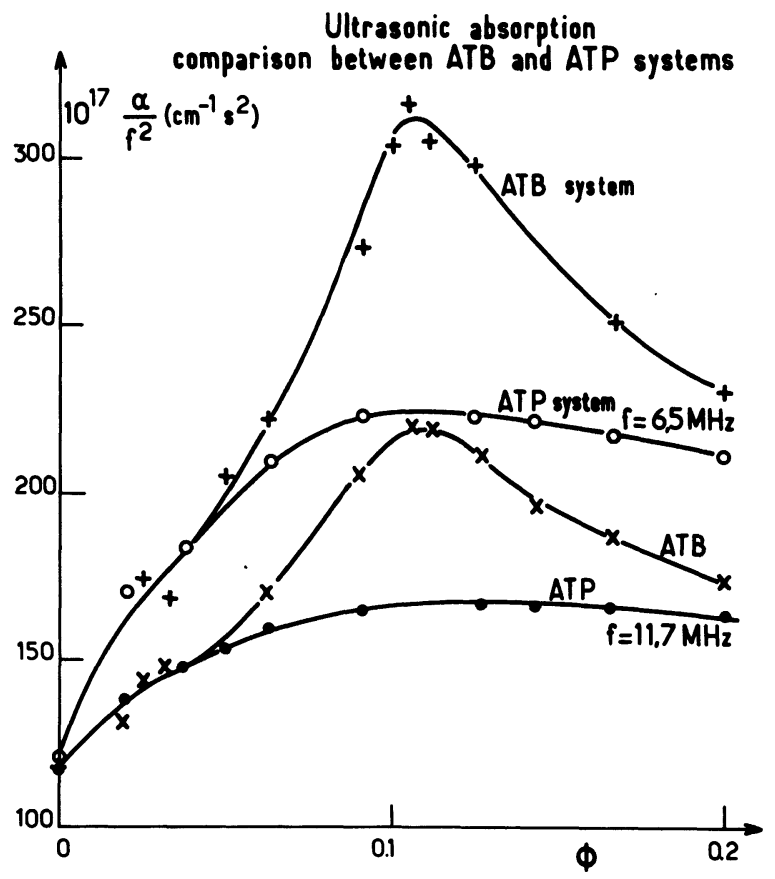

Fig. 3. - Comparison between ultrasonic absorption in ATB and ATP microemulsions for two different frequencies.

The second contribution occurs only in the range $0.05<\phi<0.2$ and goes through a maximum at $\phi \sim 0.1$. The intensity of the scattered light, the droplet diffusion coefficient, the viscosity and the electrical conductivity of the ATB system were found to behave anomalously in the same range of $\phi[1,3,16]$. As noted above, the change of electrical conductivity has been associated with a percolation effect [1], whereas the other changes have been attributed to the proximity of a critical point [16]. The viscosity results did not allow a choice between these two interpretations, which will now be considered successively in an attempt to explain the absorption maximum of the ATB system. The amplitude of this maximum will be referred to as $\Delta \alpha / f^{2}$.

For critical effects, the theoretical prediction for the exponent characterizing the frequency dependence of $\Delta \alpha / f^{2}$ varies with the ratio of the frequency $f$ of the measurements to the characteristic frequency $f^{*}$ of critical fluctuations [9]

$$
f^{*}=\frac{k T}{12 \pi^{2} \eta \xi^{3}}
$$

$\eta$ is the viscosity of the microemulsion ( $\eta \sim 2 \mathrm{cp} \mathrm{[16])} \mathrm{and} \xi$ the correlation length measured by light scattering [16]. For the present systems $\xi \sim 400 \AA$ and thus $f^{*} \sim 1.5 \times 10^{3} \mathrm{~Hz}$. This value is much smaller than the frequency at which the measurements were performed $\left(f>4 \times 10^{6} \mathrm{~Hz}\right)$. In that case $\left(f^{*}<f\right)$, Fixman's theory [9] predicts $\Delta \alpha / f^{2} \sim f^{-1.25}$, whereas the more elaborated theory of Kawasaki [10] yields $\Delta \alpha / f^{2} \sim f^{-0.96}$.

Even though our estimation of the absorption maximum $\Delta \alpha / f^{2}$ is very crude, the exponent characterizing the range of $\Delta \alpha / f^{2}$ versus $f$ is well below either of the theoretical values $(0.96$ and 1.25). This leads us to conclude that the observed ultrasonic absorption maximum cannot be due to a critical-like behaviour. This maximum can be much better understood in terms of a percolation effect.

We recall that near the percolation threshold the droplets become interconnected and form 
large clusters. The clusters are transient and this physical mechanism has been called « stirred percolation " [7]. The lifetime $\tau$ of the clusters has been estimated as $\tau \sim R^{2} / D$ where $R$ is the droplet radius and $D$ its diffusion coefficient [7]. For the ATB system, $R \sim 40 \AA, D \sim 10^{-7} \mathrm{~cm}^{2} / \mathrm{s}$ and thus $\tau_{\mathbf{R}} \sim 10^{-6} \mathrm{~s}$. One would then expect to observe a relaxational ultrasonic absorption at around $f \sim 0.1 \mathrm{MHz}$. However, the percolation effect described in reference [7] is a purely geometric one, and is not associated with volume and/or enthalpy changes. It is therefore very weakly coupled to ultrasonic waves and cannot give rise to an excess ultrasonic absorption. Likewise, the scattering of light is not large near a percolation threshold because the important fluctuations are not in the concentration, but in the connectivity [17]. Moreover, electrical percolation effects in microemulsions are certainly more complex than purely geometrical effects, since electrical percolation has never been observed for hard sphere systems [1,4] (such as the ATP system) and seems to be associated with the presence of attractive interactions. In this aspect, a site-bond percolation theory [18] is certainly needed to describe this behaviour and may also explain the relation between percolation and critical point as in gelatin gels [19].

It is also possible, as suggested by others, that open structures appear at the percolation threshold and that the droplet structure is replaced by a bicontinuous one [20]. In this picture, the transition between water-in-oil and oil-in-water microemulsions does not correspond to $\phi \sim 0.5$, but is very progressive $(0.1 \lesssim \phi \lesssim 0.9)$. The quantity of open structures at the geometrical percolation threshold is certainly related to the strength of the attractive potential : for the hardsphere-like ATP system the interface is very rigid and the structure inverts at around $\phi \sim 0.5$ without any evidence of percolation around $\phi \sim 0.1$; for the more attractiwe BCP system, open structures may appear around $\phi \sim 0.1$, but as the structure of the interface and its mean curvature are not markedly modified until $\phi \sim 0.5$ [21] the fraction of open structures should remain small until the inversion (zero curvature). The ultrasonic absorption shows only a shoulder at around $\phi \sim 0.13$ (Fig. 4), where a sharp conductivity increase is observed, and a large maximum at around

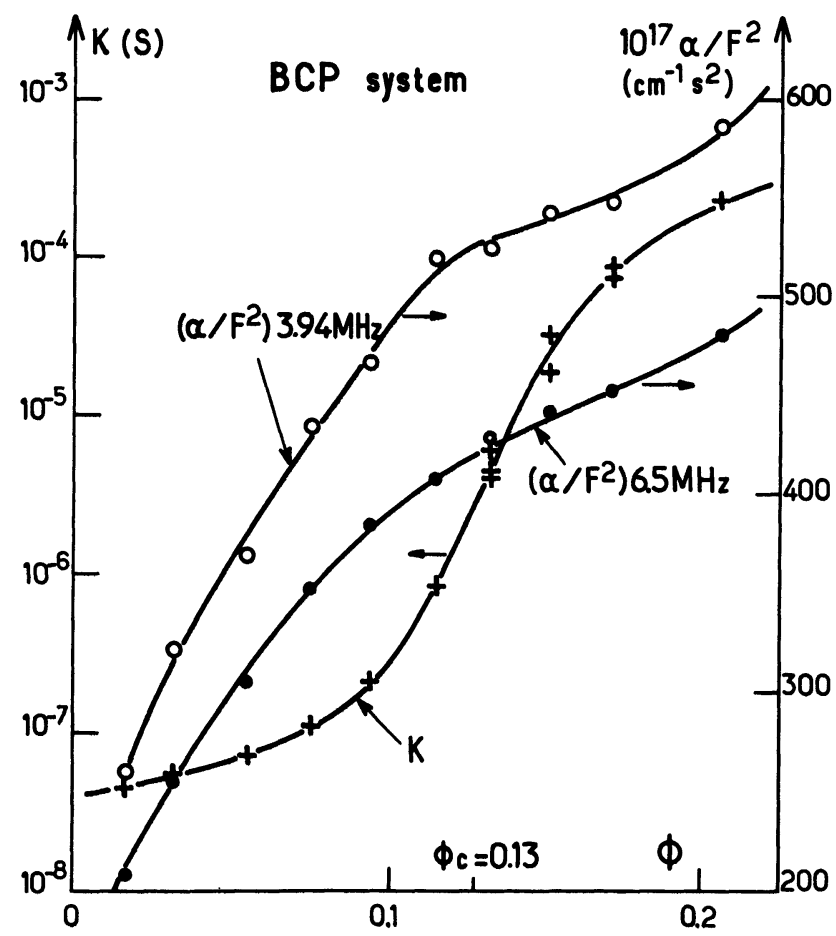

Fig. 4. - Ultrasonic absorption and electrical conductivity for BCP microemulsions versus volume fraction $\phi$ of droplets. 
$\phi \sim 0.5$. This last feature was attributed to strong changes in the exchange processes upon phase inversion $[14,15]$.

From the examination of the $\alpha / f^{2} v s . \phi$ curve for the BCP system, one cannot discard the possibility that the phase inversion contributes to the absorption of the system even at $\phi$ as low as 0.14 . The shoulder observed in the $\alpha / f^{2} v s$. $\phi$ curve would then result from the addition of this contribution to that due to the percolation effect showed by this system.

For the very attractive ATB system the fraction of open structures above the percolation threshold can be assumed to be large since attraction makes the fusion between droplets easier. The absorption maximum at around $\phi \sim 0.1$ may reflect changes in the contribution of the exchange processes similar to those in BCP system around $\phi \sim 0.5$.

Finally, let us turn to the systems which were investigated at a fixed composition as a function of temperature.

For the AOT system, figure 5 shows that the ultrasonic absorption remains nearly constant up to about $27{ }^{\circ} \mathrm{C}$, then starts increasing with temperature until the demixing temperature is reached; the lower the frequency, the larger the increase in absorption.

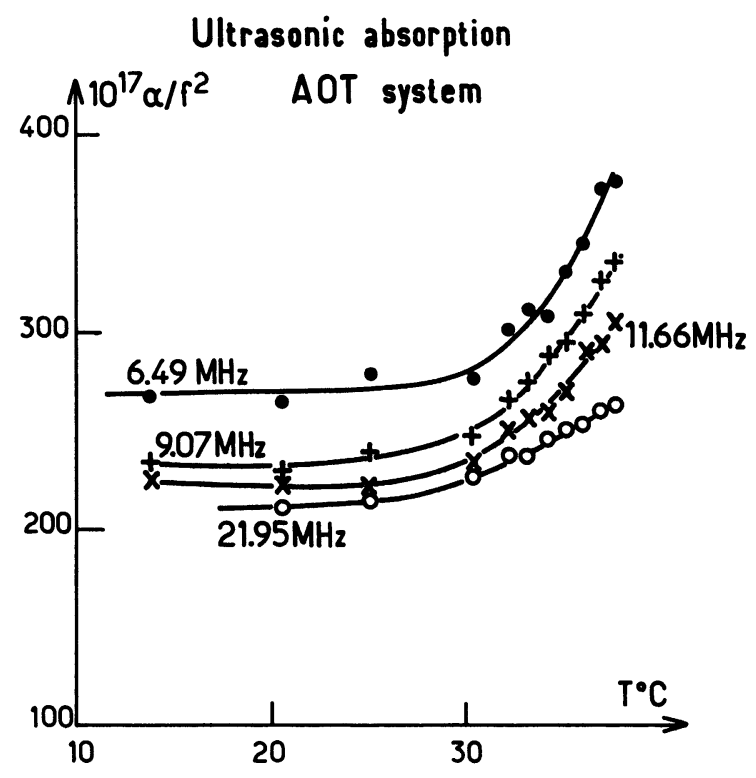

Fig. 5. - Ultrasonic absorption versus temperature in AOT system at different frequencies.

On the contrary, the absorption shows no peculiar features in the two water-external systems investigated. Thus, for the $\mathrm{C}_{12} \mathrm{E}_{6}$ system, the $\alpha / f^{2} v s$. $T$ curve shows a small decrease very similar to that found for pure water as the temperature is increased up to the demixing. (Note that the excess absorption of the $\mathrm{C}_{12} \mathrm{E}_{6}$ solution with respect to water is very small.) Likewise, the CTAB system shows only a fairly monotonic increase of $\alpha / f^{2}$ with temperature when the $\mathrm{NaBr}$ content is $1.7 \%$, that is when the system remains monophasic (see Fig. 6). The same system has also been investigated at a $\mathrm{NaBr}$ concentration of $1.88 \%$, where demixing in two phases occurs at about $24.4^{\circ} \mathrm{C}$. Again the ultrasonic absorption was found to increase smoothly with $T$ up to a temperature as close as $0.2^{\circ} \mathrm{C}$ to the demixing temperature (dotted curve in Fig. 6).

These results also go against an interpretation of the absorption increase in oil-external systems in terms of critical phenomena. Indeed, all the above systems (BCP excepted) as studied by light scattering showed similar correlation lengths. 


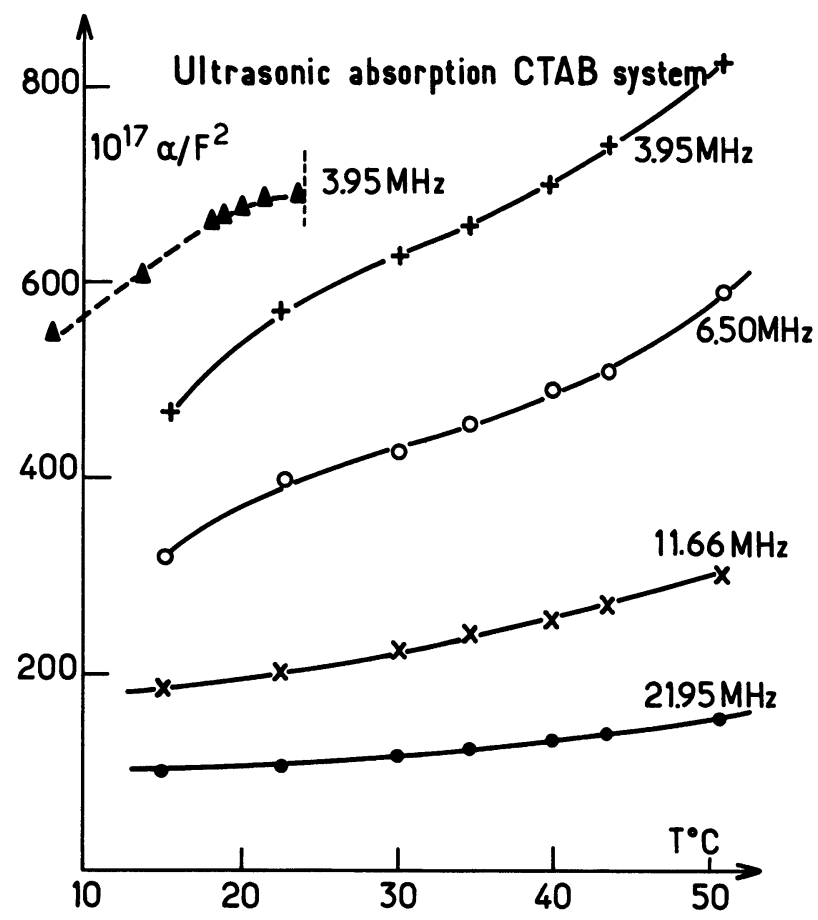

Fig. 6. - Ultrasonic absorption versus temperature in CTAB system : full lines $1.7 \% \mathrm{NaBr}$; dotted line $1.88 \% \mathrm{NaBr}$.

We can conclude that the critical contribution in the excess ultrasonic absorption seems to be quite small in all investigated systems compared to the " exchange process " contribution. (This is in contrast to binary mixtures, which show large critical absorption even far from the critical point.) In fact, the difference between oil-external and water-external systems can be better understood in term of a percolation effect and a transition towards a bicontinuous structure.

Indeed, in water-external systems the droplets are charged and this prevents collisions between them. The critical-like behaviour observed by light scattering has been attributed to a collective motion of the droplets over a certain correlation length [6,3].

On the contrary, in oil-external systems the droplets can collide, merge and then break down. During the time of droplet merging, the ions can move over large distances through water channels between droplets. The percolation occurs when the droplets' volume fraction reaches a threshold value, which corresponds to the occurrence of macroscopic aggregates of droplets. This process also gives rise to a maximum in the ultrasonic absorption (ATB system) or to an increase in the absorption (AOT system). It occurs in systems where the interfacial films are not rigid, which are usually those where attractive interactions have been observed, and which often show a criticallike behaviour of the scattered light $[1,2]$.

4. Conclusion. - In summary, the preliminary results presented in this paper provide another piece of evidence for the peculiar behaviour of some microemulsion systems. When the attraction between the microemulsion droplets is strong, the formation of open structures at the percolation threshold in oil-external systems appears to contribute to the ultrasonic absorption of the system. Although these systems and others (water-external) also exhibit critical-like behaviour, as evidenced by light scattering experiments, the ultrasonic experiments indicate that the notion of critical points has to be reconsidered in micellar systems. In particular, an increasing droplets 
polydispersity, as expected from the apparition of droplets clusters, could explain some features of the light scattering experiments [22].

More investigations are planned in order to achieve a better understanding of these systems.

\section{References}

[1] Cazabat, A. M., Chatenay, D., Langevin, D., Pouchelon, A., J. Physique-Lett. 41 (1980) L-441.

[2] Huang, J. S., Kim, M. W., Phys. Rev. Lett. 47 (1981) 1962.

[3] Dorshow, R., de Buzzaccarin, F., Bunton, C. A., Nicoli, D. F., Phys. Rev. Lett. 47 (1981) 1336.

[4] Cazabat, A. M., Langevin, D., Meunier, J., Pouchelon, A., J. Physique-Lett. 43 (1982) L-89.

[5] Fourche, G., BellocQ, A. M., BrunetTI, S., J. Colloid Interface Sci., to be published.

[6] Corti, M., Degiorgio, V., Phys. Rev. Lett. 45 (1980) 1045.

Corti, M., Degiorgio, V., Zulauf, M., Phys. Rev. Lett. 48 (1982) 1617.

[7] Laguës, M., Ober, R., Taupin, C., J. Physique-Lett. 39 (1978) L-487.

LAGUËs, M., J. Physique-Lett. 40 (1979) L-331.

[8] Setre, D., in Critical Phenomena, edited by M. S. Green (Academic Press) 1971. Mistura, L., same reference.

[9] Fixman, M., J. Chem. Phys. 36 (1962) 310 and 1957.

[10] Kawasaki, K., in Phase Transition and Critical Phenomena, edited by C. Domb and M. S. Green (Academic Press) 1976.

[11] GraciaA, A., Lachaise, J., Martinez, A., Bourrel, M., Chambu, C., J. Physique-Lett. 38 (1977) L-253.

[12] Cazabat, A. M., Langevin, D., J. Chem. Phys. 74 (1981) 3148.

[13] McLaY, W. N., J. Colloid Interface Sci. 11 (1956) 272.

[14] Lang, J., Duavanbakht, A., Zana, R., J. Phys. Chem. 84 (1980) 1541.

[15] Lang, J., Djavanbakht, A., Zana, R., in Microemulsions, edited by I. D. Robb (Plenum Press) 1982.

[16] Cazabat, A. M., Langevin, D., Sorba, O., J. Physique-Lett., à paraître.

[17] De Gennes, P. G., J. Physique-Lett. 40 (1979) L-197.

[18] Coniglio, A., Stanley, H. E., Klein, W., Phys. Rev. Lett. 42 (1979) 518.

Coniglio, A., Klein, W., J. Phys. A 13 (1980) 2775.

[19] Tanaka, T., Swislow, G., Ohmine, I., Phys. Rev. Lett. 42 (1979) 1556.

[20] Talmon, Y., Prager, S., J. Chem. Phys. 69 (1978) 2984.

[21] Dvolaitzky, M., Guyot, M., Laguës, M., Lepesant, J. P., Ober, R., Sauterey, C., Taupin, C., J. Chem. Phys. 69 (1978) 3279.

[22] Sorensen, C. M., Ackerson, B. J., Mockler, R. C., O'Sullivan, W. J., Phys. Rev. A 13 (1976) 1593. 